\title{
Anatomical Modelling of the Musculoskeletal System from MRI
}

\author{
Benjamin Gilles, Laurent Moccozet, and Nadia Magnenat-Thalmann \\ MIRALab, University of Geneva, CH-1211 Geneva, Switzerland, \\ \{gilles, moccozet, thalmann\}@miralab.unige.ch
}

\begin{abstract}
This paper presents a novel approach for multi-organ (musculoskeletal system) automatic registration and segmentation from clinical MRI datasets, based on discrete deformable models (simplex meshes). We reduce the computational complexity using multi-resolution forces, multi-resolution hierarchical collision handling and large simulation time steps (implicit integration scheme), allowing real-time user control and cost-efficient segmentation. Radial forces and topological constraints (attachments) are applied to regularize the segmentation process. Based on a medial axis constrained approximation, we efficiently characterize shapes and deformations. We validate our methods for the hip joint and the thigh (20 muscles, 4 bones) on 4 datasets: average error $=1.5 \mathrm{~mm}$, computation time $=15 \mathrm{~min}$.
\end{abstract}

\section{Introduction}

For the diagnosis, the surgical planning and the post-operative assessment of musculoskeletal disorders, the automatic segmentation of the patient musculoskeletal system is important for orthopaedists, biomechanicians and kinesiologists that would like to simulate, visualize and navigate through articulations with a minimum amount of manual tasks. As stressed by [1], 2] and [3], usual simplified models (stick-figures, muscle action lines) are not able to take into account large attachment areas, as well as global constraints such as volume preservation and non-penetration; although they are important biomechanical parameters. Therefore, the relationship between musculoskeletal dynamics and organ shapes need to be better studied through image segmentation. Magnetic Resonance Imaging (MRI) is a flexible modality for imaging both soft and bony tissues non-invasively. However, due to the large amount of textural information, noise, low-resolution, organ imbrication, and large spatial variability; automatic, fast and robust musculoskeletal segmentation is a difficult task. As a consequence, existing methods for musculoskeletal modelling are interactive [2] [4] [1] [3] and therefore time-consuming. A common approach to constrain a segmentation process is to use prior information: shape constraints rely on assumptions about surface regularity (smoothness, curvature) and variability across the population, while topological constraints exploit prior knowledge about organ interrelationships. Contrary to traditional pixel-based segmentation (e.g. level-sets) and registration (e.g. FFD) methods, these constraints can be efficiently applied 
on deformable models [5]. Physical-based simulation approaches (volumetric), including finite element or finite volume are appropriate for enforcing mechanical constraints, but their computational cost would exclude time-efficient and user-controlled segmentation. In this context, we propose to use scalable discrete deformable surfaces (simplex meshes), that can benefit from efficient geometric methods, popular in computer graphics applications.

Simplex meshes were first described by H. Delingette 6 for constrained 3D shape reconstruction and segmentation, and extended in 4D by J. Montagnat [7], with application to the heart (single model and resolution). A k-simplex mesh is defined by a set of vertices and a connectivity function (each vertex is connected to exactly $\mathrm{k}+1$ neighbors). In this paper, we use 2 -simplex meshes (dual to triangle meshes). Mesh geometric quality (uniformity of vertices repartition) and topological quality (uniformity of edge number among faces) are improved using simple topological operators. The most interesting property is its simple geometric description: three parameters (two barycentric coordinates plus the curvature) uniquely define vertex positions from their three neighbors. Based, on these parameters, smoothing and shape constraint forces are computed to regularize the segmentation. External forces are obtained through 1D registration of intensity profiles (at vertex positions and in normal direction) with generic profiles from a reference segmentation. The external force field is regularized using a local smoothing and global regularization based on the closest affine transformation.

Discrete models are commonly considered as punctual masses evolving under the Newtonian law of motion. The Newton equation leads to a first-order differential equation system relating forces to particle state (velocity and position). After forces evaluation and time discretisation, particle state can be explicitly resolved (forward Euler, Runge-Kutta) with tight time step restrictions for ensuring stability. We prefer the more stable implicit (or backward) scheme 8 9, that however requires the resolution of a large sparse linear system. To simplify force derivatives evaluation, we consider that forces have a independent anisotropic action on each particle. Non-penetration constraints are based on collision handling techniques [10. In this paper, we apply a hierarchical collision detection scheme based on 18-discrete oriented polytope(DOP) quadtrees [10]. We perform collision correction and response on particle positions, speeds and accelerations, such as in 9 .

From these background studies, we propose improvements in terms of computational speed and robustness by extending the simplex mesh framework with a multi-resolution scheme, topological constraints and medial surfaces. We show that the medial axis is suited for muscle shape analysis.

\section{Methods}

\subsection{Multi-resolution Scheme}

The use of levels of details (LODs) aims at reducing system complexity and sensitiveness to local solutions. As shape constraints spatial influence depends 
on the resolution level, the idea is to quickly propagate forces from lower resolutions to a current simulation LOD in order to get multiscale regularization. In addition, collision detection (often considered as the bottle-neck for simulation) is more efficiently performed on coarse LODs, collision response being passed to fine LODs. Indeed, exact contact computation is most of the time not relevant as fat separates organs. The tessellation of dual triangle meshes leads to a systematic and computationally efficient LOD generation scheme for simplex meshes (linear combination of vertex positions). Shape features are preserved as low level vertices are contained in higher levels. The number of vertices is quadrupled when increasing the resolution. During the simulation, forces from lower resolutions are linearly combined like vertex positions and added to current resolution forces. Even if this is not exact (vertices having moved relatively to their neighbors, since resolution increase), the estimation is still relevant, assuming that shape constraints have enforced mesh local regularity.

\subsection{Topological Constraints}

The human musculoskeletal anatomy exhibits various organs interrelationships: muscles are attached to bones, they can merge into common tendon units, fascia binds muscles and enforces frictionless contact between them. We have developed a spline-based method for generating attachment areas, so that the placement and adjustment of areas have a reduced number of degrees of freedom. Spline control points are projected onto bone surfaces, while soft-tissues vertices are attached to the spline through curvilinear coordinates. These vertices are constrained using mass modification $[8]: \mathbf{M}^{\mathbf{- 1}}=\mathbf{0}$. For individualization, we wrap splines from a generic model using spline control point barycentric coordinates on bone surface. As shown in 11, this approach is valid for most attachments as they rely on bone geometrical features. For merging deformable models, we sum forces and masses of the attached vertices. This is interesting for modelling common tendons (e.g. quadriceps femoris tendon), but also specific parts with high curvature, where smoothing forces are not appropriate (e.g. attachment between the adductor magnus and its inferior tendon). Fascia is modelled through collision handling: once generic collisions and proximities have been detected, they are subsequently used as a reference (springs) to smoothly enforce the relative position between models. In other words, we deform generic contacts.

\subsection{Radial Constraints}

Muscles generally have a smooth and tubular shape. Hence, they can be efficiently represented by an underlying piece-wise action line 1] 4], where isotonic contractions are modelled through action line shrinking/stretching, and isometric contractions through radial constraints applied to a wrapped surface. This has been applied in biomechanics for calculating joint moment arms [3]. However, muscles with large attachment areas and/or several origins/insertions require many action lines. We propose a continuous representation using medial surfaces, leading to a continuous radial regularization of the overlying surface 
and enhanced mechanisms for detecting collisions and characterizing shapes. Anchored medial surfaces (M-reps) have been successfully used in [5] 12 for constraining deformable model-based segmentation. The medial axis transform (computation of maximal ball centers and radii inside an object [13]) is an invertible transform that allows an efficient reduction of parameters dimension. Several methods have been presented for approximating the medial axis (MA), based on Voronoi diagrams, on distance maps, or on thinning.

We propose an iterative method based on forces applied to constrained deformable surfaces. Hook's spring interaction forces $f_{j}^{*}$ are applied to medial surface vertices for MA approximation ( $j$ indexes medial vertices). Reciprocally, forces $\boldsymbol{f}_{\boldsymbol{i}}$ are applied to overlying surface vertices for MA-based shape regularization ( $i$ indexes model vertices). Each model vertex $\boldsymbol{P}_{\boldsymbol{i}}$ is associated to a medial surface point $\boldsymbol{P}_{\boldsymbol{i} \perp}$ with a certain radius $r_{i}$. Radii are linearly interpolated between medial surface vertices $\boldsymbol{Q}_{\boldsymbol{j}}$ of radius $R_{j}$ to allow continuous force definition (leading to a smooth reconstructed surface from the MA). Given the weights $w_{i j}$, we have $\boldsymbol{P}_{\boldsymbol{i} \perp}=\sum_{j} w_{i j} \cdot \boldsymbol{Q}_{\boldsymbol{j}}$ and $r_{i}=\sum_{j} w_{i j} . R_{j}$. From a reference state (Fig. 1a), weights are obtained by projecting orthogonally $\boldsymbol{P}_{\boldsymbol{i}}$ onto the MA, and radii by the weighted mean $R_{j}=\sum_{i} w_{i j} . P_{i} P_{i \perp} / \sum_{i} w_{i j}$. Weights $w_{i j}$ and radii $R_{j}$ are subsequently memorized to compute radial forces for deformed states (Fig. 1b). Model forces $\boldsymbol{f}_{\boldsymbol{i}}$ are simply defined by $\boldsymbol{f}_{\boldsymbol{i}}=k .\left(1-r_{i} / P_{i} P_{i \perp}\right) \boldsymbol{P}_{\boldsymbol{i}} \boldsymbol{P}_{\boldsymbol{i} \perp}$ where $k$ is the spring stiffness (or force weight). By applying the momentum conservation law 9], we calculate the force contribution $\boldsymbol{f}_{\boldsymbol{i j}}$ for one spring $i$ at $\boldsymbol{Q}_{\boldsymbol{j}}$ such as $\boldsymbol{f}_{\boldsymbol{i j}}=-w_{i j} . \boldsymbol{f}_{\boldsymbol{i}} / \sum_{j} w_{i j}^{2}$. We average all spring forces associated to a MA vertex: $\boldsymbol{f}_{j}^{*}=$ mean $_{i}\left(\boldsymbol{f}_{\boldsymbol{i} \boldsymbol{j}}\right)$. To obtain the reference medial surface for a given model, we first construct and attach a simplex plane, based on muscle attachments(Fig. 3d). After cropping, we simulate the medial surface with radial and smoothing forces, while projecting model vertices and updating radii at each timestep. The medial surface quickly converges to the medial axis. We measure the error between the reconstructed surface from the medial axis and the desired initial model shape by calculating the mean of $\left|r_{i}-P_{i} P_{i \perp}\right|$. The average error for all tested models is $0.6 \mathrm{~mm}$. This value remains stable during the segmentation when applying radial forces to both models and axis.
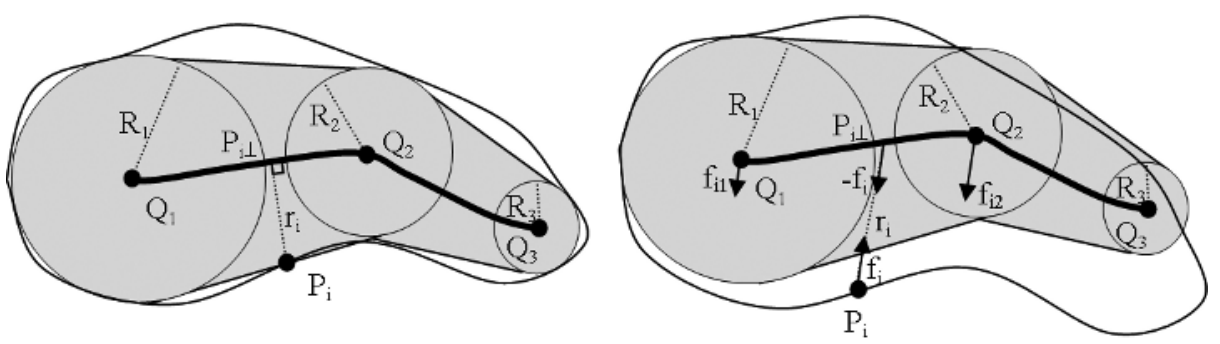

Fig. 1. Surface model and its associated medial axis in: A) A reference state; B) A deformed state; In grey, the reconstructed surface from the medial axis 
In addition, we use medial axis for collision detection: 18-DOP quadtrees hierarchies, inflated according to radii, are generated/updated for medial axis cells. Muscles vertices at a certain resolution are subsequently tested towards DOPs and projected to medial axis for the inclusion test (comparison with interpolated radii $r_{i}$ ). Independently of the model resolution, medial axis based-collision detection is around 3 times faster than surface-based collision detection. On top of MA based-collision detection, multi-resolution collision handling (section 2.1) is used to improve computation time (by a factor of 7 per LOD). Given a current resolution, we use its first coarser LOD for collision handling in order to minimize errors.

\section{Results}

\subsection{Automatic Segmentation of the Musculoskeletal System}

MRI protocol definition has been done in close collaboration with physicians from radiology and orthopaedics. The goal was to obtain images carrying sufficient information with clinically achievable protocols (fast). The final protocol was T1-weighted spin echo with TR/TE $=578 / 18 \mathrm{~ms}$, Matrix/ FOV $=512 \times 512 / 40 \mathrm{~cm}$ and slice thickness $=2 \mathrm{~mm}$ to $10 \mathrm{~mm}$ between the foot the the iliac crests (five series in total, $\sim 150$ slices, $\sim 30$ minutes).

Based on interactive segmentation, we have reconstructed a generic model of the hip and the thigh, composed of a skin, 20 muscles, 4 bones and the corresponding attachment splines. After topological optimization, the different LODs (3 for soft-tissues, 4 for bones) and medial surfaces were generated. The final surface model (highest resolution) is composed of 71328 vertices for muscles and 85100 vertices for bones. Alternatively, muscles can be represented by their medial surfaces (1 resolution, 3821 vertices) with an average error of $0.6 \mathrm{~mm}$. The compression factor (parameter dimension reduction) is equal to $(3 \times 71328) /(4 \times 3821)=14$ (3 dimensions for model points, 4 for axis points, including radii).

Before automatic individualization, the generic model is coarsely initialized using ten manually placed landmarks corresponding to anatomical landmarks, and using thin-plate-spline (TPS) interpolation. After this step, bones are deformed (Euler implicit integration) from the coarse level to the fine level using multiresolution internal forces (shape and smoothing constraints), non-penetration constraints (multi-resolution collision handling) and intensity profile-based external forces. Muscles are subsequently individualized (Fig. 2) as follows: A) attachment splines are initialized on bone surfaces from their generic barycentric coordinates; B) a skeleton-driven deformation algorithm [14] (skinning) is applied to generic muscles and medial surfaces according to joint angles; $\mathrm{C}$ ) soft-tissues/medial surfaces are deformed using internal forces (radial/shape and smoothing constraints), proximity constraints (deforming contacts), and skin surface matching (gradient-based external forces); D) soft-tissues are deformed from the coarse level to the fine level using internal forces, proximity/nonpenetration constraints and intensity profile-based external forces. The best 
intensity profile size and resolution have been experimentally defined from interactively segmented models by minimizing excursions. Being more variable, the external part of the profile is less relevant (thus shorter). The intensity profile search depth decreases from $20 \mathrm{~mm}$ to $5 \mathrm{~mm}$ during segmentation (Fig. 2). We found that normalized cross-correlation is the most robust metric for intensity profile similarity measure. The affine regularization contribution decreases from $100 \%$ to $20 \%$. For fine resolutions, internal forces from coarse levels are propagated (global/local constraints) as presented in 2.1. Muscle shape constraints are derived from their medial surfaces with generic radii and weights. However, radii are updated for fine levels to give more freedom to surfaces. Radial forces are applied simultaneously to surfaces and to medial axis with an equal contribution. For coarse levels, generic proximity constraints are applied (deforming contacts). These constraints are released for the finest level, where multi-resolution medial axis-based collision detection is applied to allow surface sliding.

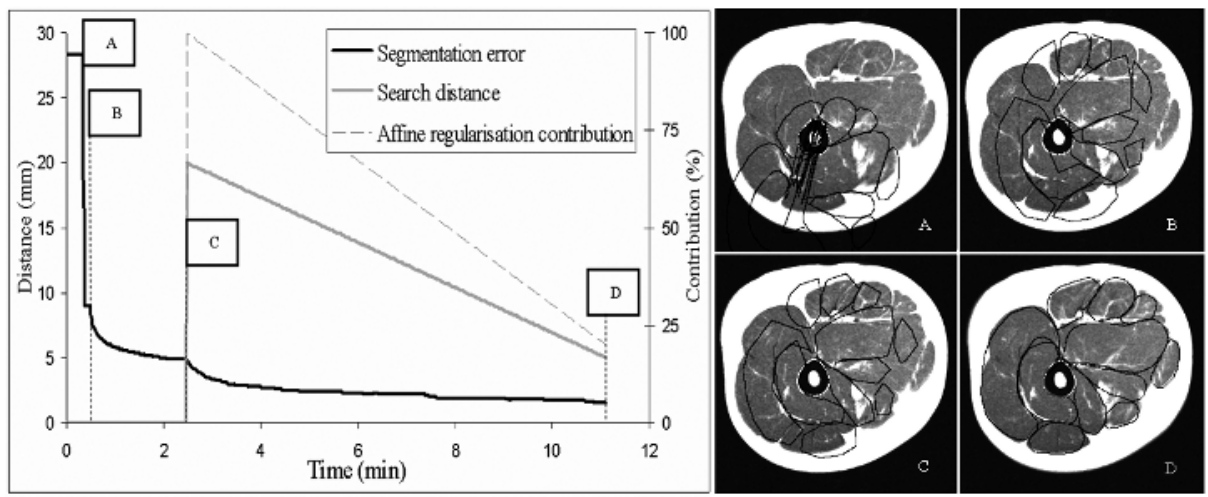

Fig. 2. Automatic muscle segmentation process and result on a sample slice: A) Generic model with wrapped attachments; B) Skeleton-driven model initialization; C) Segmentation using internal forces and skin matching (min. resolution); D) Final segmentation using external forces (max. resolution)

During the segmentation, it is possible to interactively place constraint points on the images, to get a faster matching and a more accurate segmentation. Using our collision detection scheme, deformable models are forced to include or exclude these constraint points. In addition to the generic model, this method has been applied by a medical student to segment accurately 4 datasets from 4 different healthy subjects ( 2 females and 2 males). We compared automatically segmented models to these reference models (Fig. 2). The average distance (std. dev.) was $1.25 \mathrm{~mm}(1 \mathrm{~mm})$ for bones and $1.7 \mathrm{~mm}(1.8 \mathrm{~mm})$ for muscles. The overall computation time for the automatic method is around $15 \mathrm{~min}$ on a standard PC, for which $3 / 4$ of the time is spent on external forces computation. Computation time for each timestep, including visualization, is around $0.5 \mathrm{sec}$. 


\subsection{Shape Analysis}

Musculoskeletal shape characterization is important for anthropometric comparison between individuals, and deformation analysis (temporal and longitudinal studies). For this purpose, our method registers anatomical features through shape and topological constraints. High-level descriptors such as the medial axis convey more information than local descriptors (curvature). Muscle thickness can be simply analyzed through medial axis radii comparison, as shown in Fig. 3b. Using geodesic distances to attachments, we compute normalized coordinates $X$ and $Y$ along medial surfaces, from which a thickness profile can be extracted (maximum radius in $Y$ direction). For some muscles showing thickness steep changes, tendons lengths (which is an important biomechanical parameter) can be automatically extracted (Fig. 3f).

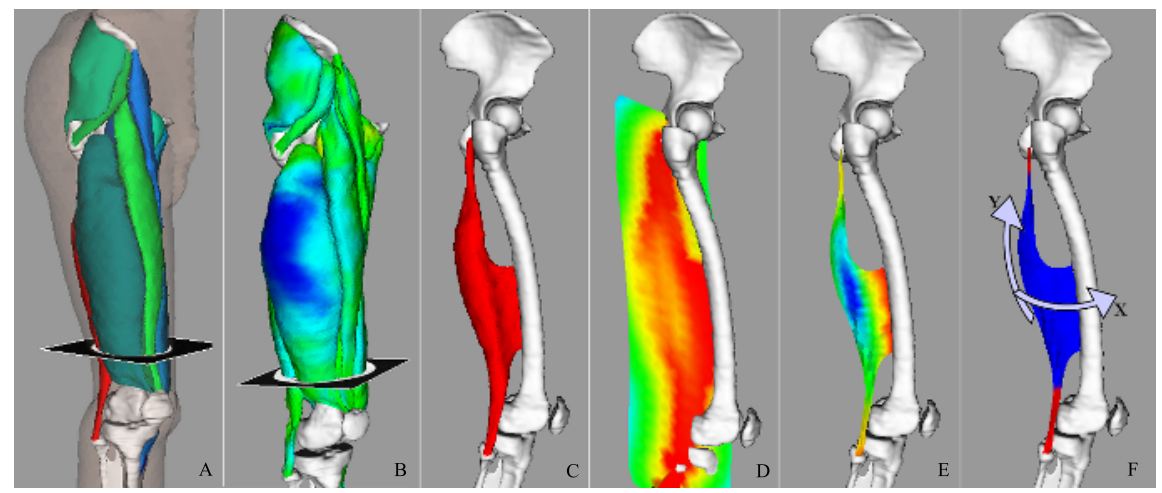

Fig. 3. A) Reconstructed generic model; B) Muscle thickness comparison with an individualized model (blue: $\geq 5 \mathrm{~mm}$; red: $\leq 5 \mathrm{~mm}$ ); C) Biceps femoris generic model; D) Initialized medial axis (blue: max radius, red: min radius); E) Medial axis after cropping and fitting; F) Tendon selection (in red) after thickness profile analysis along Y

\section{Discussion and Future Work}

Prior low-level (e.g. curvature) and high-level (e.g. medial axis) shape information and topological relationships (e.g. proximities, attachments) are relevant for musculoskeletal modelling, and complexity can be efficiently decreased using a multi-resolution approach for force and contact computation. By constraining the problem, our goal is to get a fast and accurate segmentation from a minimum amount of information: we want to extend our previous work on bone motion extraction from real-time dynamic MRI [15] (6 low resolution slices) by extracting soft tissue deformation. We believe that we can get a higher accuracy through a deeper study of intensity profile forces (combination of several metrics, weighting according to profile relevance, etc.) and by adding statistical constraints applied to high-level descriptors (e.g. medial axis radii) according to joint angles. The 
next step will be to relate these descriptors with dynamic (e.g. moment arms) and physiological parameters (e.g. muscle activation from EMG). This will provide useful information for validating functional biomechanical models.

Acknowledgments. This work is supported by CO-ME (Computer Aided and Image Guided Medical Interventions) project funded by Swiss National Research Foundation. We would like to thank Dr. Kolo-Christophe, Dr. N'Guyen and Dr. Sadri from the Geneva University Hospital for their collaboration.

\section{References}

1. Teran, J., Sifakis, E., Blemker, S., Ng-Thow-Hing, V., Lau, C., Fedkiw, R.: Creating and simulating skeletal muscle from the visible human data set. IEEE TVCG 11 (2005) 317-328

2. Ng-Thow-Hing, V.: Anatomically-based models for physical and geometric reconstruction of humans and other animals. Ph.D. Thesis, Department of Computer Science, University of Toronto (2000)

3. Blemker, S.S., Delp, S.L.: Three-dimensional representation of complex muscle architectures and geometries. Annals of Biomedical Eng. 33 (2005) 661-673

4. Aubel, A., Thalmann, D.: Interactive modeling of the human musculature. Proc. of Computer Animation (2001)

5. Terzopoulos, D., Witkin, A., Kass, M.: Symmetry-seeking models and 3d object reconstruction. International Journal of Computer Vision 1 (1987) 211-221

6. Delingette, H.: General object reconstruction based on simplex meshes. International Journal of Computer Vision 32 (1999) 111-146

7. Montagnat, J., Delingette, H.: 4d deformable models with temporal constraints: application to 4d cardiac image segmentation. MIA 9 (2005) 87-100

8. Baraff, D., Witkin, A.: Large steps in cloth simulation. Proc. of SIGGRAPH98, Computer Graphics 32 (1998) 106-117

9. Volino, P., Magnenat-Thalmann, N.: Implementing fast cloth simulation with collision response. Proc. of the Int. Conference on Computer Graphics (2000) 257-268

10. Teschner, M., Kimmerle, S., Zachmann, G., Heidelberger, B., Raghupathi, L., Fuhrmann, A., Cani, M.P., Faure, F., Magnenat-Thalmann, N., Strasser, W.: Collision detection for deformable objects. Proc. of Eurographics State-of-the-Art Report (2004) 119-135

11. Kaptein, B.L., VanDerHelm, F.C.T.: Estimating muscle attachment contours by transforming geometrical bone models. Journal of Biomechanics 37 (2004) 263-273

12. Pizer, S.M., Fletcher, P.T., Joshi, S., Thall, A., Chen, J.Z., Fridman, Y., Fritsch, D.S., Gash, A.G., Glotzer, J.M., Jiroutek, M.R., Lu, C., Muller, K.E., Tracton, G., Yushkevich, P., Chaney, E.L.: Deformable m-reps for 3d medical image segmentation. International Journal of Computer Vision 55 (2003) 85-106

13. Blum, H.: A transformation for extracting new descriptors of shape. Models for the Perception of Speech and Visual Form (1967)

14. Kalra, P., Magnenat-Thalmann, N., Moccozet, L., Sannier, G., Aubel, A., Thalmann, D.: Real-time animation of realistic virtual humans. Computer Graphics and Applications 18 (1998) 42-56

15. Gilles, B., Perrin, R., Magnenat-Thalmann, N., Vallée, J.P.: Bones motion analysis from dynamic mri: acquisition and tracking. Proc. of MICCAI'04 2 (2004) 942-949 